\title{
TICS, COMUNICACIÓN Y PARTICIPACIÓN CIUDADANA EN LOS PLANES DE GOBIERNO ABIERTO DE ARGENTINA, COSTA RICA Y MÉXICO
}

\author{
ICTS, COMMUNICATION AND CITIZEN PARTICIPATION IN THE \\ OPEN GOVERNMENT PLANS OF ARGENTINA, COSTA RICA AND \\ MEXICO
}

\author{
Luisa Eugenia Ochoa Chaves \\ Universidad de Costa Rica, Costa Rica \\ Francisco García García \\ Universidad Complutense de Madrid, España
}

\section{Paula Monge Espinoza}

Universidad de Costa Rica, Costa Rica

Autor para correspondencia: Luisa Eugenia Ochoa Chaves, email: luisa.ochoa@ucr.ac.cr

\section{Resumen}

Este trabajo indaga las formas en que las Tecnologías de la Información y la Comunicación (TICs) propician o no una comunicación gubernamental más horizontal en la era de la cultura participativa y digital. Para ello, se analiza el contenido de los compromisos relacionados con el desarrollo y/o mantenimiento de las TICs de los tres primeros planes acción de Gobierno Abierto de México, Costa Rica y Argentina. Se caracterizan los compromisos en términos de su aporte a la transparencia, la participación ciudadana y la colaboración —los tres pilares de Gobierno Abierto- los niveles de participación y el tipo de plataformas tecnológicas que proponen para potenciar la participación ciudadana. Los resultados sugieren que aún estamos en la primera etapa del proceso de implementación de un modelo de Gobierno Abierto, caracterizada por compromisos en el ámbito de la transparencia y que se limitan a acciones informativas. Sin embargo, se pueden apreciar una transición a una comunicación más participativa: en el diseño de las plataformas tecnológicas, en la inclusión de las TICs en estrategias que combinan acciones virtuales y presenciales para fomentar la participación ciudadana, y en el ciclo completo de diseño y evaluación de las políticas públicas.

Palabras clave: Tecnologías de la información y la comunicación, TICs, participación ciudadana, comunicación, Gobierno Abierto

Global Media Journal México 17(32), enero - junio 2020, pp. 19-41. 


\begin{abstract}
This paper investigates the ways in which Information and Communication Technologies (ICTs) promote or not a more horizontal government communication in the era of participatory and digital culture. For this, content analysis is used to characterize the commitments related to the development and/or maintenance of ICTs of the first three action plans of the Open Government for Mexico, Costa Rica and Argentina. The commitments are analyzed in terms of their contribution to transparency, citizen participation and collaboration - the three pillars of Open Government-, the levels of participation, and the type of technological platforms that they propose to enhance citizen participation. The results suggest that we are still in the first stage of the process of implementing an Open Government model, characterized by commitments in the field of transparency and that are limited to informative actions. However, a transition to a more participatory communication can be seen in the design of technological platforms, in the inclusion of ICTs in strategies that combine virtual and face-to-face actions to promote citizen participation, and in the complete cycle of design and evaluation of public policies.
\end{abstract}

Keywords: Information and communication technologies, ICTs, citizen participation, communication, Open Government.

Recibido: 26/01/2020

Aceptado: 28/03/2020

\section{Introducción}

Asistimos a un momento histórico de insatisfacción con respecto del funcionamiento de la democracia asociada a la falta de fluidez comunicativa entre representantes y representados (Martínez et al., 2005). La desafección política de la ciudadanía se manifiesta de diversas maneras: retraimiento de los partidos, abstencionismo electoral, desconfianza en las instituciones representativas $\mathrm{y}$ de sus intermediarios, etc. $\mathrm{Y}$, aunque estas actitudes no implican un rechazo de la democracia como sistema de gobierno, sí señalan distanciamiento entre los gobiernos y la ciudadanía, "de ahí que la apelación a las supuestas virtudes regenerativas de la tecnopolítica, de la promesa de una democracia vigorizada por las NTIC, parezcan más producto del deseo y la ilusión que de la reflexión matizada" (Martínez et al., 2005, p. 23).

La idea de que las Tecnologías de la Información y la Comunicación (TICs) vendrían a modificar las relaciones entre gobiernos y ciudadanía se inspira en el supuesto de que estamos ante sociedades que se mueven por fuerzas productivas como la información y el

Global Media Journal México 17(32), enero - junio 2020, pp. 19-41. 
conocimiento (Mattelart \& Multigner, 2007).

El Gobierno Abierto propone una nueva forma de gestión pública en la que se crean alianzas entre ciudadanos y gobiernos a todos los niveles para lograr los mejores resultados. Además, incluye promesas asociadas al desarrollo de las TICs, y dentro de estas, proyecta cambios en las relaciones entre actores sociales como es el caso de la interacción entre gobiernos y ciudadanía, especialmente desde su dimensión participativa.

En este contexto, se analizan los Planes de Acción de Gobierno Abierto de tres países de América Latina desde la participación ciudadana, entendida como una relación comunicativa entre gobiernos y ciudadana y que está mediada el desarrollo de (TICs).

La contribución del trabajo se expone mediante tres ejes: primero, comprender cómo entienden los gobiernos a las TICs, y cómo consideran que su incorporación puede aportar a la participación ciudadana. Segundo, contribuir a problematizar conceptos y promesas asociadas al Gobierno Abierto y las TICs a partir de evidencia empírica y el análisis de contenido. Y tercero, aportar conocimientos sobre los procesos de producción de TICs, para así facilitar la participación ciudadana en un momento histórico marcado por una desafección política de la ciudadanía y los debates sobre la necesidad de democracias más participativas y deliberativas.

El análisis de los compromisos incluidos en los Planes de Acción de Gobierno Abierto es una forma de abordar, desde la investigación empírica, cuál el camino que está tomando la producción de TICs para la participación ciudadana.

\section{La promesa: gobierno abierto y TICs}

El gobierno abierto surgió en medio de demandas sociales cada vez más complejas y variadas. Por una parte la crisis de la democracia representativa (Martínez et al., 2005), que se manifiesta en el aumento del abstencionismo y la disminución de la potencia de los partidos políticos; y, por otra, de una ciudadanía que demanda participación, que usa las TICs, y que aprovecha los modelos de interacción derivados de la cultura digital (trabajo colaborativo y en red).

Los tres elementos en los que se basa el gobierno abierto son: transparencia, participación y colaboración (Corojan y Domínguez, 2011). Transparencia en el acceso a la información y a los datos abiertos, para que así la ciudadanía pueda valorar y participar en los asuntos públicos. Participación en el involucramiento activo de la ciudadanía en la conformación de políticas públicas, para la mejora de la eficacia del gobierno y de la calidad de sus decisiones. Y colaboración en el diseño, implementación y seguimiento de políticas públicas. Sin transparencia la ciudadanía no dispondrá de la información suficiente para valorar y participar en los asuntos públicos, sin participación en la toma de decisiones no habrá

Global Media Journal México 17(32), enero - junio 2020, pp. 19-41. 
motivación para colaborar, y sin colaboración de la ciudadanía en la gestión pública se pondrían en riesgo los gobiernos y sus democracias.

Por otra parte, si el gobierno abierto es la propuesta desde lo gubernamental, desde la sociedad civil está el movimiento de datos abiertos, que busca que los datos públicos que generan los gobiernos puedan ser accesibles, reutilizables y comprensibles a la ciudadanía. El movimiento está compuesto por organizaciones sociales, la academia, periodistas de datos y profesionales de las ingenierías y del software libre (Damasco, 2017), que intercambian iniciativas y conocimientos inspirados en elementos de la cultura colaborativa.

Actualmente hay un desplazamiento hacia el eje de Estado abierto, que implica ver cómo el modelo de gobierno abierto se extiende "a todos los niveles y poderes del Estado" (Vilches, 2016), aunque en este artículo vamos a centrar el análisis en la perspectiva de gobierno abierto.

El gobierno abierto tiene antecedentes en el gobierno electrónico, desde el momento en que se comenzó a utilizar la Web como un canal paralelo para el acceso a los servicios por parte de la ciudadanía (Concha y Naser, 2012). De manera que se comienza a configurar una oferta de servicios digitales que se remiten a un modelo de comunicación en una sola vía: llenado y envío de formularios electrónicos o de transacciones completas, como obtención de visas y pasaportes, certificados de nacimiento y defunción, pago de multas e impuestos, etcétera (Concha y Naser, 2012, p. 13).
Desde la comunicación, lo que confiere un valor distintivo a la democracia como forma de gobierno es la exigencia ética de que las decisiones políticas (las que afectan a toda la comunidad) se tomen en condiciones de publicidad que permitan la presentación, discusión y clarificación de las distintas opciones existentes sobre aquello que haya que decidir (Martínez et al., 2005, p. 23). Es por esto que interesa analizar la participación ciudadana como una relación comunicativa en la que diversos actores sociales interactúan con los gobiernos, proceso sobre el que opera la democracia, y en el cual las TICs pueden influir en el cambio del modelo de comunicación de una perspectiva vertical o unidireccional hacia una más participativa e interactiva.

El gobierno electrónico plantea una comunicación que ha dejado de lado la potencialidad de la tecnología desde su dimensión interactiva y participativa, y que sigue la perspectiva unidireccional y vertical de la comunicación tradicional de masas:

El sistema de comunicación de la sociedad industrial se centraba en los medios de comunicación de masas, caracterizados por la distribución masiva de un mensaje unidireccional de uno a muchos. La base de la comunicación de la sociedad en red es la web global de redes de comunicación horizontal que incluyen el intercambio multimodal de mensajes interactivos de muchos a muchos, tanto sincrónicos como asincrónicos (Castells, 2008, p. 7).

Global Media Journal México 17(32), enero - junio 2020, pp. 19-41. 
Aunque el gobierno abierto de alguna manera da continuidad al Gobierno Electrónico, se diferencia de este al estar influenciado por la convergencia de medios y la cultura participativa.

En los últimos tiempos, la convergencia de medios se ha visto impulsada por ofrecer a los usuarios nuevas formas de consumir contenidos y de una interactividad que permite una comunicación one to one, one to many o many to many, en donde los roles emisor y receptor se intercambian constantemente como adelantaron Marshall McLuhan y Barrington Nevitt (1972), afirmando que la tecnología electrónica permitiría al consumidor asumir simultáneamente los roles de productor y consumidor de contenidos (Sánchez e Ibar 2015, p. 88).

La cultura participativa, que en un primer momento describe la producción cultural y las interacciones sociales de las comunidades de fans, ha "evolucionado y ahora hace referencia a un conjunto de grupos distintos que utilizan la producción y distribución mediática para satisfacer sus intereses colectivos" (Jenkins et al., 2013, p. 24). En este contexto de intercambio de roles entre productor y consumidor de información, la ciudadanía convertida en usuarios de tecnologías cobra un lugar central para el desarrollo del potencial democrático a partir de una cultura de participación.

Lo que plantea la necesidad de superar "la falta de disposición de las administraciones al diálogo con el ciudadano y al desaprovechamiento de los recursos que ofrecen para iniciar un intercambio abierto $y$ enriquecedor que permita pasar del egovernment al we-government" (Ure, 2015, p. 248). Dentro de este enfoque el gobierno abierto es entendido como un nuevo paradigma que potencia y actualiza las promesas del $e$ government, y lo supera al prometer un modelo de comunicación bidireccional.

Algunos ejemplos son las listas de distribución electrónica, las redes electrónicas, los portales electrónicos de organizaciones sociales, los foros virtuales, blogs, los grupos de discusión y comunidades temáticas (PetrizzoPáez, 2005, p. 95). Estos son instrumentos comunicativos en internet que sirven para que la ciudadanía se implique en el debate de asuntos públicos (Martínez et al., 2005, p. 25), mecanismos a través de los cuales la ciudadanía puede ejercer el control sobre los recursos y las decisiones públicas que afectan su vida.

Las TICs pueden servir de soporte para la información y facilitar la interacción de las personas usuarias con los contenidos y con otras personas usuarias. De estas posibilidades tecnológicas se desprenden las expectativas que dan inicio a la renovación de la comunicación política democrática (Martínez et al., 2005, p. 26) que estarían asociadas 1) con el aumento de la información, recursos y documentos relacionados con asuntos cívicos; 2) con el acceso a expertos o especialistas; 3) con las acciones ciudadanas provenientes de los

Global Media Journal México 17(32), enero - junio 2020, pp. 19-41. 
escenarios presenciales o virtuales; 4) con el intercambio con una mayor cantidad de actores sociales o ciudadanos no cercanos; 5) con los nuevos escenarios de discusión ciudadana (que bien pueden ser grupos pequeños o grandes de discusión alrededor de determinados temas), y 6 ) con el mayor contacto con modelos ciudadanos provenientes de otros lugares.

A partir de estos elementos nos preguntamos: ¿cuáles son las iniciativas que se incluyen en los planes de acción de gobierno abierto de México, Costa Rica y Argentina para fomentar la participación ciudadana con la ayuda de las TICs?

\section{Contexto: gobierno abierto en Argentina, Costa Rica y México}

La popularización de la perspectiva de gobierno abierto se da con la creación de la Open Government Partnership (OGP) en el 2011, en el marco de la Asamblea LXVI de las Naciones Unidas en Nueva York. Esta inicia con ocho países miembros y tiene ahora 75 países participantes. La OGP es la instancia gubernamental internacional que promueve y da seguimiento a los compromisos de acción, en coordinación con instancias nacionales.

Para ser miembro de esta organización los países participantes deben firmar la Declaración de Principios sobre gobierno abierto y con ello adquirir compromisos concretos: 1) un plan de acción nacional elaborado mediante proceso de consulta participativa con la ciudadanía; 2) un informe de evaluación por un panel de expertos independientes, y 3) contribuir a la promoción de gobierno abierto a través del intercambio de mejores prácticas, conocimientos y asistencia técnica.

México fue uno de los miembros fundadores de la OGP y consolidó desde el 2013 el Secretariado Técnico Tripartita como órgano de deliberación y decisión donde participan sociedad civil y gobierno. Durante los años 2016-2018 trabajó en su III plan de acción, con compromisos alineados con la Agenda 2030 de Desarrollo Sostenible de Naciones Unidas. También ha avanzado en la construcción de metodologías de implementación, seguimiento y monitoreo de compromisos adoptados (“Gobierno abierto en México", 2017).

Durante el 2018, 10 organizaciones renunciaron al Secretariado Técnico Tripartita tras denunciar a la administración de Enrique Peña Nieto por no actuar frente al caso de espionaje ilegal de diferentes oficinas gubernamentales a personas investigadoras $\mathrm{y}$ defensoras de la salud en dicho país ("Sociedad civil denuncia espionaje”, 2017).

"La violencia contra periodistas en esta administración ha sido históricamente la más alta y el periodo electoral de 2018 ha sido el más mortal, con más de 100 políticos asesinados. No hay avances en la denuncia de espionaje digital ilegal a

Global Media Journal México 17(32), enero - junio 2020, pp. 19-41. 
Ochoa Chaves, García García y Monge Espinoza 25

pesar de los constantes reclamos de las víctimas, sociedad civil y relatores internacionales de libertad de expresión de la ONU y la OEA (Núcleo de la Sociedad Civil para el Gobierno Abierto en México, 2018).

Para marzo de 2019, México reactiva el proceso nacional de la Alianza para el gobierno abierto con un comunicado conjunto del nuevo gobierno de Andrés Manuel López Obrador y el Núcleo de Organizaciones de la Sociedad Civil (NOSC). El proceso se reanuda con la creación del Comité Coordinador para la construcción colaborativa del Cuarto Plan de Acción 20192021 de México ante la OGP (Alianza para el Gobierno Abierto México, 2019) .

Por otra parte, Costa Rica ha avanzado en la creación de un índice de transparencia del sector público, en la formulación e implementación de leyes y políticas relacionadas con el acceso a la información pública, la apertura de datos, la participación ciudadana y la lucha contra la corrupción. También se han generado procesos de capacitación para funcionarios públicos $\mathrm{y}$ la difusión $\mathrm{e}$ implementación de las políticas ya aprobadas, por ejemplo, la difusión de la Política de Participación Ciudadana del Poder Judicial (Comisión Nacional de Gobierno Abierto, 2015).

Los avances se han concretado, entre otras acciones, en el Decreto de Transparencia y Acceso a la Información Pública y el fortalecimiento de la Ley 8220 de Protección al
Ciudadano ante el Exceso de Requisitos y Trámites Administrativos. Además, representantes de todos los poderes de la república suscribieron un Convenio de Estado Abierto para avanzar a nivel país en las áreas mencionados anteriormente. Para dar seguimiento a estas medidas está la Comisión Nacional por un Gobierno Abierto, en la que participan miembros de la empresa privada (Unión Costarricense de Cámaras y Asociaciones del Sector Empresarial Privado), la academia (Consejo Nacional de Rectores) y la sociedad civil (Abriendo Datos y Costa Rica Íntegra).

Hay un claro interés gubernamental en impulsar la agenda de gobierno abierto, al ser este uno de los elementos que son tenidos en cuenta en el proceso de adhesión de Costa Rica a la Organización para la Cooperación y el Desarrollo Económicos (OCDE).

Por último, Argentina se integró de manera formal a la Alianza en noviembre de 2012, al igual que México y Costa Rica, y se encuentra en la etapa de desarrollo de su $3^{\circ}$ Plan de Acción 2017-2019. Algunos avances en el desarrollo del gobierno abierto en Argentina son la aprobación de la Ley 27078 Argentina Digital, que tiene el fin de alcanzar la universalidad en el acceso y eliminar las desigualdades en el ámbito de la comunicación y la información; la sanción de la Ley 27275 de Derecho de Acceso a la Información Pública, cuyo objetivo es garantizar el ejercicio de este derecho; el Plan de Apertura de Datos, en el cual

Global Media Journal México 17(32), enero - junio 2020, pp. 19-41. 
los ministerios, secretarías y organismos desconcentrados y descentralizados se comprometen a tener disponible en formatos abiertos ciertos activos de información; la creación de Andino, una tecnología que facilita la publicación de datos en formato abierto; y el lanzamiento de la Plataforma de Consulta Pública, que representa un canal de diálogo entre el gobierno y la ciudadanía (Borrmann, 2017).

En 2018, se realizó un diagnóstico que evalúa el progreso en la implementación de los compromisos del $3^{\circ}$ Plan del Gobierno Abierto de Argentina, que incluye 18 compromisos con nivel alto de cumplimiento, 15 con nivel medio y 11 con nivel bajo de cumplimiento de los hitos planteados (OGP Argentina, 2018).

En cada uno de los tres países se presentan contextos diferentes en los cuales se ha dado la incorporación del gobierno abierto. En México, luego de un período de crisis en los diálogos entre gobierno y sociedad civil aparece una nueva oportunidad bajo el nuevo gobierno de López Obrador. En Costa Rica se han creado diferentes instancias para dar seguimiento a los acuerdos generados en los planes de gobierno abierto y se ha avanzado en la formulación de directrices para la apertura y acceso a la información. En Argentina, al igual que en Costa Rica, se han creado normativas y planes para establecer procedimientos frente al acceso a la información pública y la apertura de datos abiertos. De estos contextos es importante tener en cuenta la articulación de diferentes actores alrededor de la generación de los planes de gobierno abierto.

\section{Metodología}

Esta es una investigación descriptiva a partir del análisis de contenido de los compromisos de nueve planes de gobierno abierto disponibles para estos tres países de América Latina. Se analiza de forma cualitativa cada uno de los compromisos y se verifica la presencia de temas, palabras o conceptos con relación a tres ejes de análisis: relación del compromiso con el modelo de gobierno abierto, los niveles de participación y TICs que proponen. Una vez clasificados se hace el análisis de contenido cuantitativo para establecer frecuencias según cada eje de análisis.

Se utiliza el análisis de contenido como una técnica de investigación que permite formular a partir de ciertos datos inferencias reproducibles y válidas que puedan aplicarse en su contexto (Krippendorff, 1997, p. 28). El análisis de contenido aplicado es de tipo cualitativo para verificar la presencia de temas, de palabras o de conceptos en un contenido y cuantitativo porque cuantifica los datos, establece frecuencias y comparaciones a partir de la aparición de los elementos de significación (Gómez Mendoza, 2012, p. 4).

Se analiza el contenido manifiesto de la comunicación incluida en los compromisos de gobierno abierto. Si bien se aborda brevemente el contexto externo a los compromisos en la descripción del proceso de gobierno abierto en cada país, el centro del estudio está en el análisis interno de los documentos, procurando destacar

Global Media Journal México 17(32), enero - junio 2020, pp. 19-41. 
su sentido y características fundamentales en función de la pregunta de investigación.

Los tres países de América Latina fueron seleccionados por presentar indicadores altos de penetración de Internet en su área.

En el sur, Argentina presenta los indicadores más altos de penetración de Internet, seguido de Paraguay (89.6\%) y Uruguay (88.2\%). En Centroamérica, Costa Rica tiene el indicador más alto seguido de Panamá (69.1\%). En las estadísticas disponibles en el sitio web Miniwatts Marketing Group México (2018) se incluye en Centroamérica y se ubica en el tercer puesto de penetración, luego de Panamá y antes de El Salvador (50.3\%). Hay que señalar que en Centroamérica se encuentra una media de $59.7 \%$, mientras que en Suramérica es de $71.5 \%$.

Tabla 1

Contexto de penetración de Internet en Costa Rica, Argentina y México

\begin{tabular}{llll}
\hline & Costa Rica & Argentina & México \\
\hline Población & $4,953,199$ & $44,688,864$ & $130,759,074$ \\
\% penetración Internet & $86.9 \%$ & $93.1 \%$ & $65.3 \%$ \\
Conexiones cada 100 habitantes & 156 & 125.6 & 79.8 \\
Usuarios de telefonía móvil & $8,300,000$ & $50,409,800$ & $88,797,186$
\end{tabular}

Fuente: Tabla elaborada a partir de datos publicados en los sitios web Miniwatts Marketing Group (2018) y Wikipedia (2018).

La Alianza para el Gobierno Abierto comienza en el 2011, y desde esa fecha cada país incluido en la investigación ha presentado tres planes de acción. Un plan de acción de gobierno abierto es el conjunto de compromisos concretos $y$ realizables, se trata de la hoja de ruta para que los gobiernos incorporen y concreten esta perspectiva en su quehacer, y su construcción implica etapas de consulta pública, jornadas abiertas y mesas de trabajo. En esta investigación son fuentes documentales que permiten analizar el desarrollo de TICs para la participación ciudadana, por lo que, una vez seleccionados los países, se procede al análisis de contenido de estos planes, documentos elaborados por la Alianza para el Gobierno Abierto México (2011; 2013, 2016), Presidencia República de Costa Rica $(2013 ; 2015 ; 2017)$ y Presidencia de la Nación de Argentina (2013; 2015; 2017).

Procedimiento de análisis: 1) se revisaron los 182 compromisos de los nueve planes de gobierno abierto analizados para determinar si había relación directa con la creación y/o mantenimiento de TICs, y si eso estaba presente en el compromiso se incluía en la siguiente etapa

Global Media Journal México 17(32), enero - junio 2020, pp. 19-41. 
de análisis; 2) se identificaron 99 compromisos relacionados con el desarrollo o mantenimiento de TICs en los nueve planes analizados, y 3) se caracterizaron los 99 compromisos según las siguientes variables a) relación con el modelo de gobierno abierto, b) niveles de participación ciudadana a los cuales se orientan, y c) soluciones tecnológicas para el cumplimiento de los compromisos y su relación con la participación.

Es importante indicar que no todos los documentos o planes cuentan con el mismo nivel de detalle sobre las plataformas, para facilitar esta tarea se buscó en Internet información complementaria: páginas web o noticias sobre el gobierno abierto de cada país.

Tabla 2

Cantidad de compromisos de gobierno abierto por país

\begin{tabular}{lccccccccc}
\hline & \multicolumn{3}{c}{ Plan 1 } & \multicolumn{3}{c}{ Plan 2 } & \multicolumn{3}{c}{ Plan 3 } \\
\hline País & Total 1 & TIC 2 & TIC \% & Total & TIC & TIC \% & Total & TIC & TIC \% \\
Argentina & 19 & 6 & 31.6 & 15 & 12 & 80.0 & 44 & 24 & 54.5 \\
Costa Rica & 23 & 11 & 47.8 & 18 & 3 & 16.7 & 12 & 6 & 47.8 \\
México & 18 & 8 & 44.4 & 26 & 24 & 92.3 & 7 & 5 & 44.4 \\
Total & 60 & 25 & 41.7 & 59 & 39 & 66.1 & 63 & 35 & 55.6 \\
\hline
\end{tabular}

(1) Muestra la cantidad de compromisos totales que tiene cada plan de acción.

(2) Muestra la cantidad de compromisos relacionados con la creación o mejoramiento de TICs.

\section{Gobierno abierto y TICs}

La tabla 2 muestra la cantidad total de compromisos que tiene cada plan de acción y los compromisos directamente relacionados con TICs.

Lo primero que se puede observar es que la presencia de TICs en los tres planes de gobierno abierto se ha sostenido entre $40 \%$ y $60 \%$. En el primer plan de acción hay un porcentaje de compromisos relacionados con la producción de TICs que se encuentran entre 30\% y 50\%. En el segundo plan de acción, Argentina y México tienen un incremento de presencia de tecnologías que supera $80 \%$ de compromisos, sin embargo, Costa Rica tiene una disminución de $17 \%$; en su lugar hay más compromisos relacionados con la creación o fortalecimiento de normativa y legislación entorno a la transparencia, el acceso a la información, la lucha contra la corrupción y apertura de datos.

En el tercer plan, los tres países se encuentran alineados con la cantidad de compromisos relacionados con la producción de TICs, los cuales oscilan entre $45 \%$ y $55 \%$. Eso

Global Media Journal México 17(32), enero - junio 2020, pp. 19-41. 
quiere decir que en la mitad de los compromisos de gobierno abierto hay alguna referencia a la implementación de plataformas tecnológicas para la gestión y publicación de información.

Aunque han habido fluctuaciones en la cantidad de compromisos relacionados con tecnología en los planes de acción analizados, es evidente la importancia de las TICs en la forma en la que se implementa los planes de acción, siendo fundamental en la mitad de los compromisos propuestos.

\section{Figura 1}

Porcentaje de compromisos relacionados con TICs

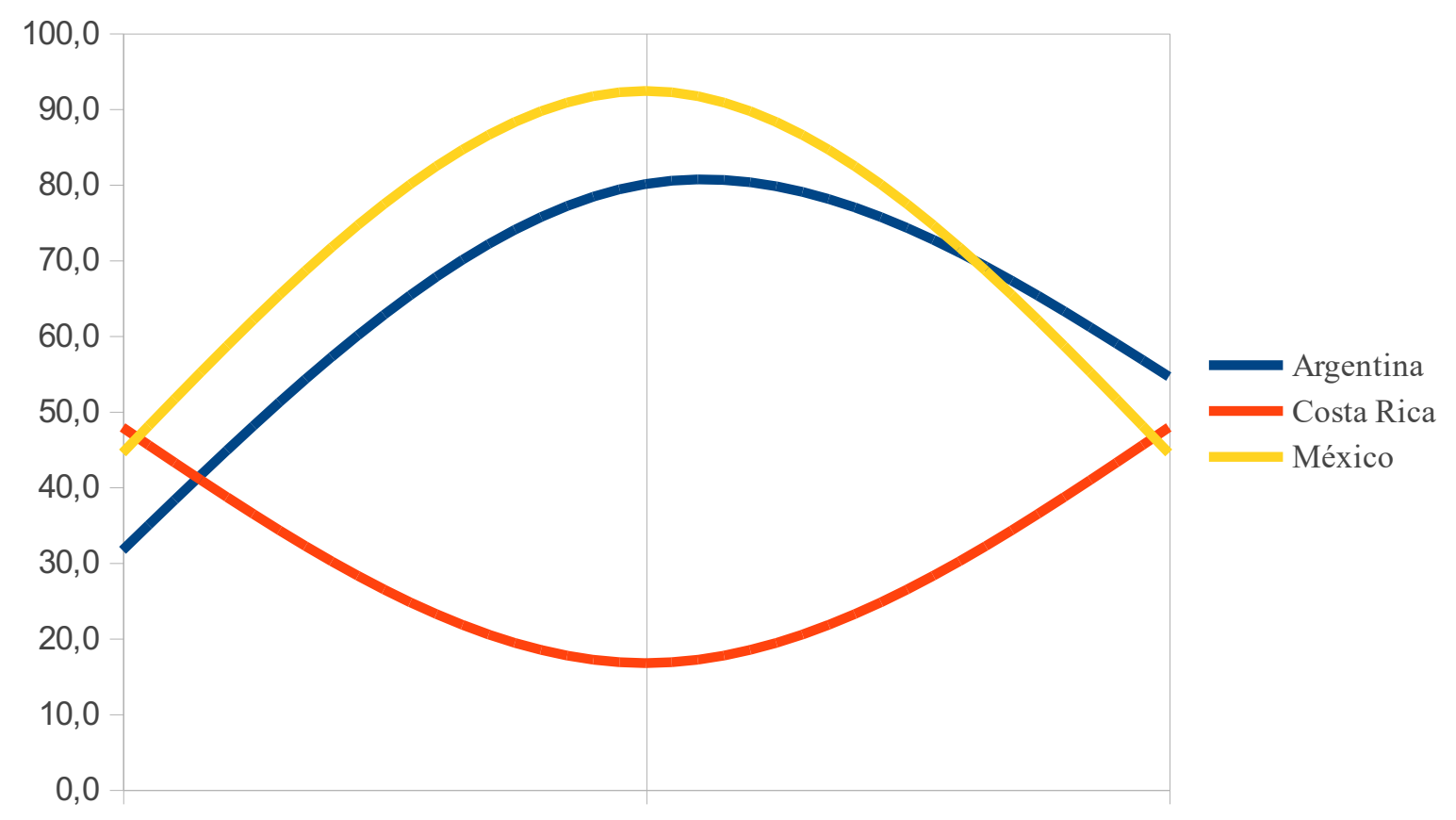

TICs para la transparencia, participación y colaboración

A continuación, la cantidad de compromisos según los principios básicos del gobierno abierto: transparencia, participación $\mathrm{y}$ colaboración. Además, se ha incluido un tema emergente a partir del análisis de los datos, compromisos dedicados a la TICs en la gestión pública, que agrupa los compromisos que no están directamente relacionados con los tres ejes anteriores y cuyo objetivo es el mejoramiento de los servicios gubernamentales destinados a la ciudadanía mediante el uso de las TICs. Estos compromisos muestran la continuidad de la perspectiva de Gobierno Electrónico.

Se observa un énfasis en los compromisos de transparencia, se trata de propuestas que buscan generar dispositivos para recolectar, sistematizar la información y luego ponerla a Global Media Journal México 17(32), enero - junio 2020, pp. 19-41. 
disposición de la ciudadanía. México y Costa Rica tiene tantos compromisos en Argentina presentan una mayoría de transparencia como en gestión pública. compromisos en transparencia, mientras que

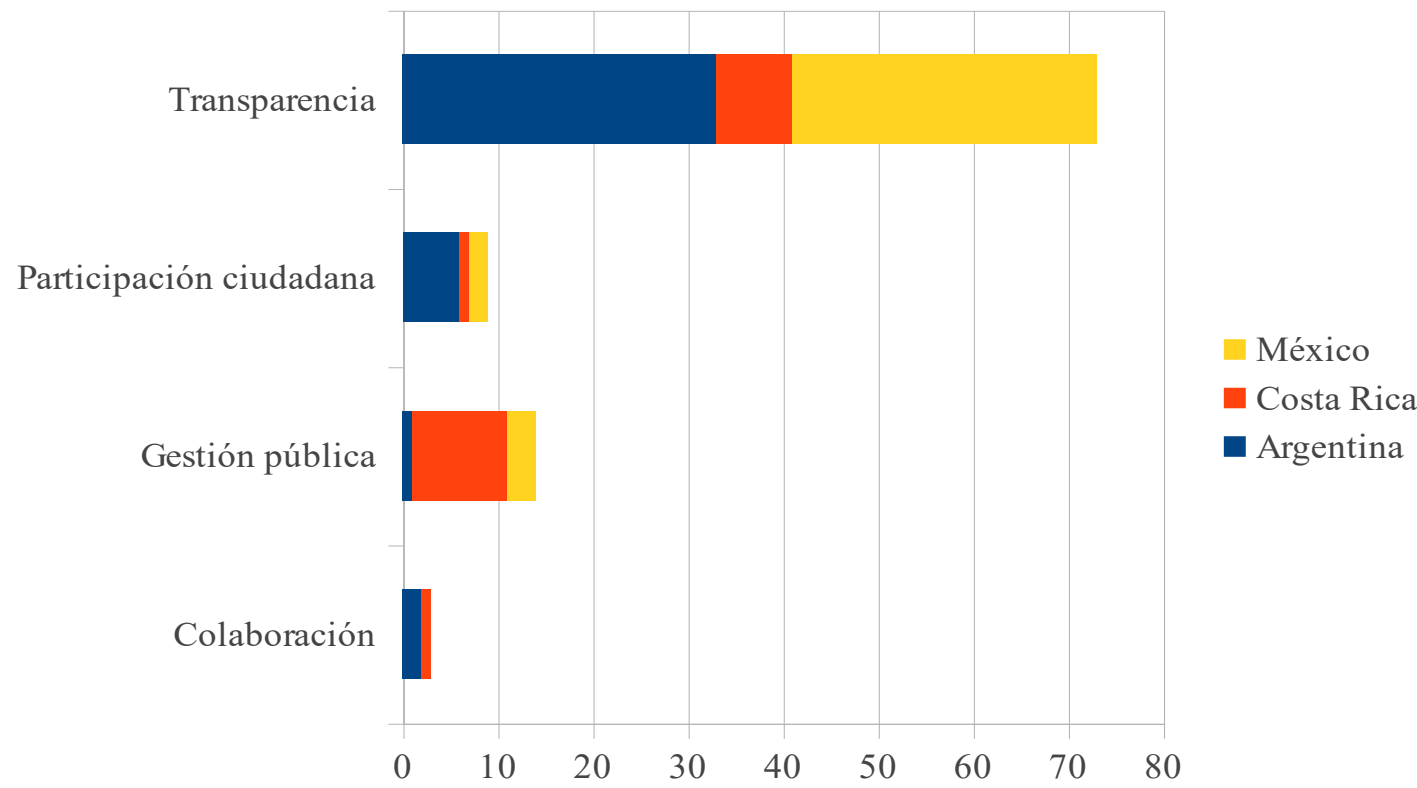

Figura 2

Compromisos según principios de gobierno abierto

Los compromisos de gobierno abierto de los tres países clasificados en el eje de transparencia plantean iniciativas relacionadas con TICs que proponen acciones de comunicación en una sola vía y centradas en la información, y acciones que implican diversos grados de participación, estas últimas son iniciativas tecnológicas que involucran de alguna forma a las personas usuarias.

Si bien las iniciativas de transparencia relacionadas con la publicación de datos sobre obras, presupuesto público y registro de personas - son un avance al brindar acceso a la información pública de forma proactiva, el modelo de comunicación que se propone sigue siendo unidireccional. En este caso, la ciudadanía es un actor pasivo que aporta solo en la medida en que sus datos son parte de la oferta de datos disponible en las plataformas tecnológicas de las instituciones públicas.

En cuanto a los compromisos que implican algún tipo de participación, destacan las posibilidades del monitoreo de políticas públicas y las plataformas para la gestión de solicitudes de acceso a la información. En estos casos, las TICs son plataformas que van más allá de la incorporación de las tecnologías a la gestión y prestación de servicios públicos, y de

Global Media Journal México 17(32), enero - junio 2020, pp. 19-41. 
la perspectiva instrumental de la ciudadanía como cliente.

Tabla 3

Comunicación en los compromisos de transparencia

\begin{tabular}{lcl}
\hline Comunicación & Síntesis de compromisos \\
\hline Información & 1. $\begin{array}{l}\text { Publicación de información y estadísticas sobre temas especializados, } \\
\text { en algunos casos con datos georeferenciados, sobre proyectos de } \\
\text { infraestructura, obras públicas, educación pública, justicia, políticas } \\
\text { públicas culturales, equidad de género, entre otros. }\end{array}$ \\
& 2. $\begin{array}{l}\text { Publicación periódica y actualizada sobre inversiones, finanzas públicas } \\
\text { y transferencias de recursos públicos. }\end{array}$ \\
3. $\begin{array}{l}\text { Crear registros de personas en diferentes tipos de situación como } \\
\text { personas desaparecidas o beneficiarios de programas sociales. }\end{array}$ \\
4articipación
\end{tabular}

Por otra parte, un conjunto de compromisos de gobierno abierto que han sido agrupados en gestión pública tiene que ver con la gestión de los servicios que brindan los gobiernos a la ciudadanía tales como servicios digitales y de realización de trámites. Esto podría interpretarse como una herencia directa de las promesas pendientes del gobierno electrónico que consiste en utilizar las TICs para mejorar el servicio y la descentralización de territorios.

Con respecto a los compromisos relacionados con la participación ciudadana estos se presentan en tres modalidades: 1) diseño de plataformas virtuales mediante procesos participativos, que proponen metodologías que integran de manera conjunta actores de la sociedad civil, academia, expertos y representantes del gobiernos en la producción de TICs; 2) producción de plataformas participativas que incluyen espacios virtuales como portales web y redes sociales para la colaboración entre organizaciones de sociedad civil y el Estado, y 3) promoción de la participación ciudadana en el ciclo de las políticas públicas a través de encuentros presenciales e instrumentos virtuales, por ejemplo, el Compromiso 17 del $1^{\circ}$ Plan de Acción de Costa Rica, que habla de "abrir espacios de participación para que los ciudadanos puedan comunicar sus requerimientos, contribuyan en la formulación y evaluación de políticas públicas y proyectos" (Presidencia República de Costa Rica, 2013).

Las participación se relaciona con la implementación de plataformas interactivas,

Global Media Journal México 17(32), enero - junio 2020, pp. 19-41. 
como la que se menciona en el Compromiso 31 de la Guía Joven del $3^{\circ}$ Plan de Acción de Argentina, que consiste en "generar una plataforma interactiva centralizada en donde cualquier persona pueda relevar, informar, detectar y retroalimentar políticas públicas destinadas a jóvenes de entre 15 y 24 años en todo el territorio nacional" (Presidencia de la Nación Argentina, 2017).

El eje de colaboración en los compromisos de gobierno abierto investigados se presenta en tres compromisos relacionados con la colaboración mediante la participación en laboratorios de innovación, que invitan a la ciudadanía a participar en la toma de decisiones mediante plataformas o repositorios de proyectos tecnológicos en donde se les insta a compartir y colaborar con sus proyectos de tecnología.

\section{Niveles de participación en los compromisos de gobierno abierto}

En la interacción entre gobiernos y ciudadanía hay distintos niveles de participación, por lo que es importante determinar es si existen los canales adecuados para todos ellos, teniendo en cuenta que "la mera información es solo un peldaño o un prerrequisito para la participación, que en definitiva significa entregarle poder a la ciudadanía" (Concha y Naser, 2012, p. 14).

Para profundizar en el análisis de la participación ciudadana en el marco del gobierno abierto se usó el modelo de niveles de participación en el marco del gobierno abierto elaborado por Cruz-Rubio (2015, p. 22), en el cual se definen cinco niveles: informar, consultar, involucrar, colaborar y dar el poder de tomar decisiones.

Destaca que 'informar' sea el eje que agrupa más compromisos de gobierno abierto, 72 de los 99 compromisos estudiados, lo que indica que el aporte de las TICs a la participación ciudadana está limitada a un primer nivel de participación, y reitera que la implementación del gobierno abierto está restringido a una comunicación en una sola vía con la ciudadanía, que la coloca en un lugar pasivo del proceso comunicativo.

Aunque en la categoría 'informar' se incluye la perspectiva de datos abiertos, ocho compromisos indican de forma explícita el acceso a la información pública en formatos abiertos, y no se cuenta con evidencia que permita indicar mayores niveles de participación a partir de la reutilización de los datos. Los compromisos que se vinculan con datos abiertos son iniciativas relacionadas con: contrataciones públicas, decisiones legislativas (Senado o Congreso abierto) y Tribunales de Justicia (justicia abierta). De este tipo de compromisos hay cuatro en Argentina, uno en Costa Rica y tres en México.

En la categoría 'consultar' hay 15 compromisos relacionados con la posibilidad de que las personas ciudadanas puedan enviar información al gobierno mediante las TICs, es decir, que la comunicación no es sólo 
unidireccional, sino que puede ser bidireccional.

\section{Figura 3}

Cantidad de compromisos de gobierno abierto según niveles de participación

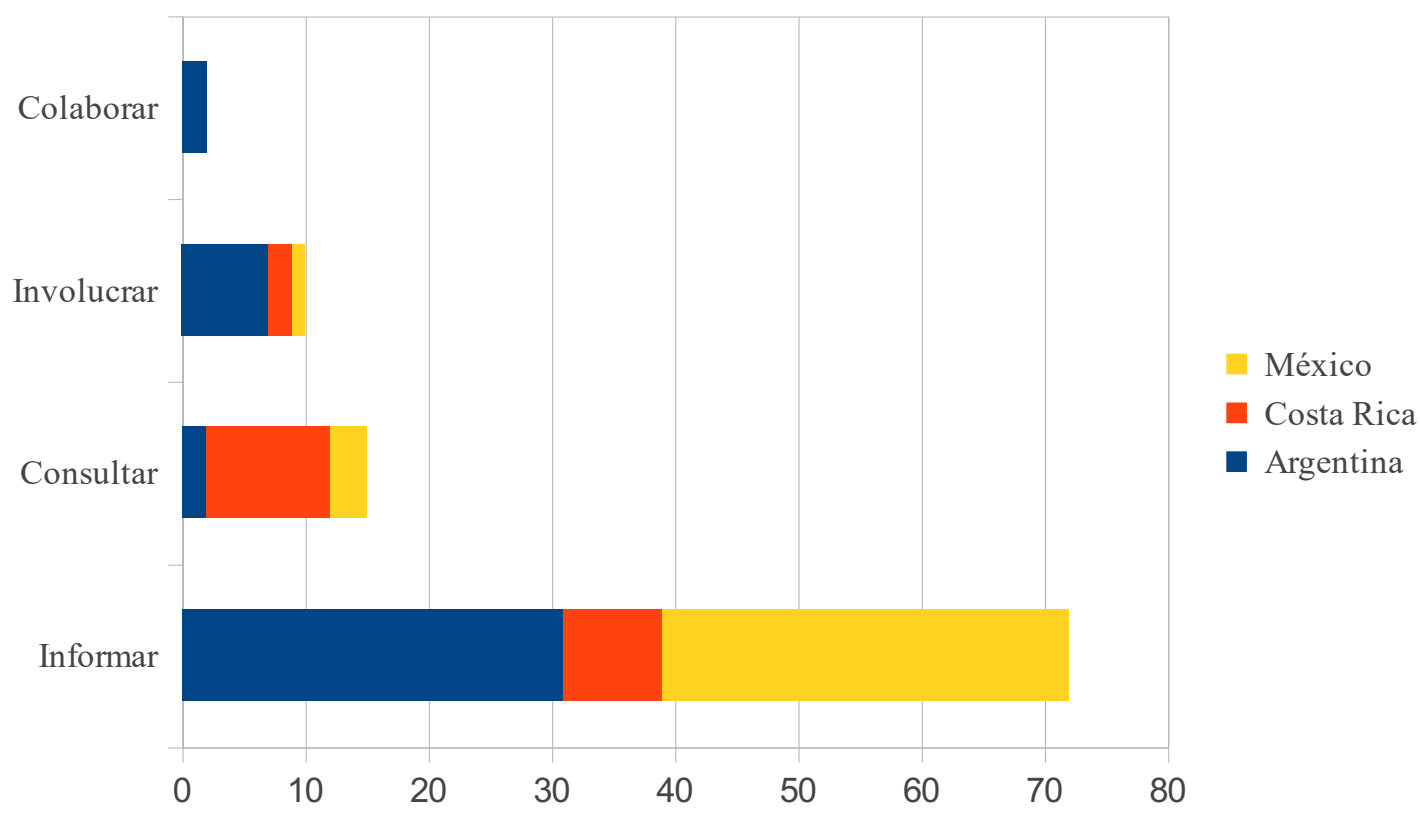

Algunos ejemplos son los compromisos relacionados con fortalecer los portales gubernamentales, como www.gob.mx o www.gob.go.cr, para convertirlos en herramientas interactivas, implementar electrónicamente acceso a la información de justicia, sistemas únicos de compra, plataformas de seguridad ciudadana para dar aviso a la policía, y ampliar los servicios en las Ventanillas Electrónicas de Servicio (VES). En muchos de estos casos el tipo de interacción propuesta está marcada por la realización de un trámite o un servicio mediado por las tecnologías.

En la categoría 'involucrar' aparecen 10 compromisos relacionados con el trabajo conjunto con organizaciones de sociedad civil, en este grupo hay un énfasis en promover la participación ciudadana en el ciclo de las políticas públicas a través de una combinación de acciones presenciales y virtuales. Ejemplos relacionados con el involucramiento de la ciudadanía son los compromisos de gobierno abierto Guía Joven, para incluir a jóvenes de entre 15 y 24 años en la construcción de las políticas de juventud, y Participación Ciudadana en Políticas Culturales del Programa Casas de la Historia y la Cultura del Bicentenario, los dos de Argentina; o el compromiso Laboratorio de Innovación para Ciudades Sostenibles e Inclusivas, de Costa Rica, para crear espacios participativos y de trabajo colaborativo para el diseño, implementación y monitoreo de proyectos en las ciudades. Es importante indicar que, de los 10 compromisos de involucramiento de la ciudadanía, siete son de Argentina. 
En la categoría 'colaborar' hay dos compromisos, el primero relacionado con una plataforma online para monitorear la ejecución programática y presupuestaria del Plan para la Prevención, Asistencia y Erradicación de la Violencia contra las Mujeres 2017-2019, en Argentina; el segundo propone una red colaborativa entre organizaciones de la sociedad civil y el Estado en la ciudad argentina de Corrientes, y plantea el desarrollo de una plataforma virtual de soporte de la red integrada por un portal, espacio en redes sociales y una aplicación móvil.
En conclusión, predomina un nivel de participación informativo. En los compromisos de mayores niveles de participación hay iniciativas de apertura de espacios virtuales donde se pueden compartir necesidades y proyectos, y la utilización de las tecnologías para el monitoreo de políticas públicas y la creación de redes entre Estado y sociedad civil. Finalmente, se puede observar la ausencia del nivel de participación que implica la toma de decisiones, es decir que los compromisos de gobierno abierto no incluyen iniciativas como votaciones o procesos de deliberación vinculantes con el diseño y ejecución de políticas públicas.

\section{Figura 4}

Tipos de plataformas incluidas en los compromisos

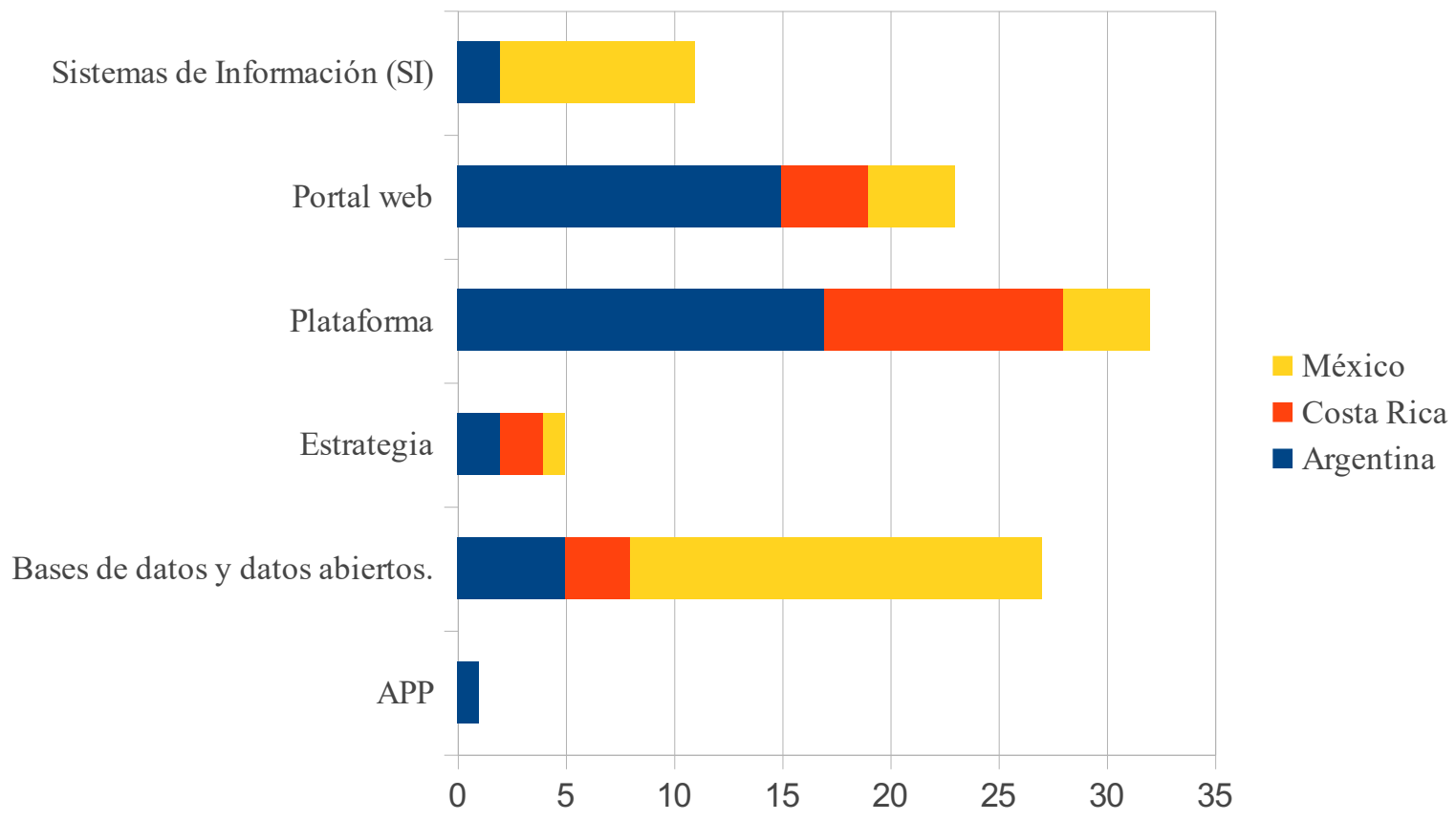

Global Media Journal México 17(32), enero - junio 2020, pp. 19-41. 


\section{Tecnologías para el gobierno abierto}

En este apartado se quiere indagar sobre la materialidad que tiene que ver con cuál es la plataforma tecnológica que soporta la oferta de contenidos. Se entiende por plataformas tecnológicas los desarrollos informáticos que permiten la organización y publicación de contenidos, el despliegue y distribución de la información a través de enlaces, y la interacción entre personas usuarias. Pueden ser portales web, aplicaciones móviles, sistemas de información, foros virtuales, entre otros.

Como se observa en el gráfico, en México predominan bases de datos y sistemas de información, lo que sugiere que el país está en una etapa de digitalización, centralización y actualización de sistemas de gestión de información pública.

Este tipo de plataformas tecnológicas facilitan la gestión de la información, su clasificación, ordenamiento y disponibilidad, sin embargo, no necesariamente están relacionadas con la publicación de datos o información para la ciudadanía y pueden quedarse simplemente a un nivel de uso al interno de las instituciones.

Los sistemas de información aparecen relacionados con los compromisos de transparencia, preocupados por dar cuenta de los procesos de contratación pública, obra pública y beneficiarios de políticas públicas.

En la categoría 'bases de datos y apertura de datos' se agrupan los compromisos de gobierno abierto que señalan la creación de bases de datos y la publicación de datos abiertos. Estas plataformas están relacionadas con la publicación de información estadística, contratos aprobados, sistemas de becas, evaluaciones de impacto ambiental, indicadores de cambio climático, reportes de personas desaparecidas, directorios de las organizaciones de la sociedad civil, y en general, información que el Estado ya tiene y que puede poner a disposición de la ciudadanía de forma proactiva en internet.

En los compromisos en los que se mencionan de forma explícita la apertura de datos se propone dar a conocer información siguiendo estándares de datos abiertos. De lo anterior se destaca la generación de estrategias en las que se incluye o menciona la necesidad de automatizar o implementar herramientas digitales en procesos de gestión pública y los compromisos que señalan directamente la necesidad de incorporar la georeferenciación en los datos a presentar en portales web o plataformas.

Por otra parte, los portales web, las estrategias o las plataformas tienen mayor presencia en Costa Rica y Argentina y remiten a compromisos que plantean tecnologías más participativas.

Con respecto a la categoría 'plataforma', hay una indefinición del tipo de artefacto tecnológico a diseñar, se trata de una forma abierta, que parece relacionada con la creación de plataformas para la información -

Global Media Journal México 17(32), enero - junio 2020, pp. 19-41. 
plataformas con funciones especializadas que integran la retroalimentación de las personas usuarias para cumplir sus objetivos- y plataformas de monitoreo y participación en políticas públicas.

Plataformas de información para: a) transparentar procesos gubernamentales, como normas oficiales, gasto público, procesos de selección de funcionarios públicos (magistrados), proyectos prioritarios del Ministerio de Modernización; b) mapear actores y lugares, como directorios informativos de instituciones públicas e información acerca de villas y asentamientos, y c) ofrecer servicios a la ciudadanía, como información para contribuyentes, servicios y trámites en línea sobre registros sanitarios y estado de los procesos acusatorios.

Plataformas que hacen cosas como, por ejemplo, un sistema integral de información sobre el consumo de agua en México, una plataforma para la disminución y prevención de la delincuencia recibiendo colaboración de la sociedad civil en Costa Rica (denuncias en línea) o una plataforma para la vinculación tecnológica virtual que busca articular necesidades $\mathrm{y}$ capacidades institucionales para el desarrollo de proyectos tecnológicos en Argentina.

Plataformas de monitoreo y diseño de políticas públicas: hay dos compromisos relacionados con las plataformas para monitorear planes y programas relacionados con la protección de los derechos de las mujeres. Otros temas tienen que ver con políticas de desarrollo productivo y sus beneficiarios, monitoreo de la implementación de planes y acciones de gobierno abierto, políticas públicas culturales y políticas destinadas a jóvenes.

Los portales web dan cuenta de dos tendencias, 1) la función de ventanilla única para la realización de trámites en línea, que está vinculada con la descentralización de la administración pública y es una herencia del gobierno electrónico y 2) la creación de portales de datos abiertos en cada país relacionado con el movimiento datos abiertos.

\section{Conclusiones}

En este artículo se problematizan las promesas asociadas a las Tecnologías de la Información y la Comunicación (TICs) para la participación ciudadana, mediante el análisis de contenido de los planes de acción de gobierno abierto de Argentina, Costa Rica y México.

Desde la comunicación se indaga por la participación ciudadana, en tanto la relación comunicativa que vincula a los gobiernos con la ciudadanía, y viceversa, resulta fundamental porque soporta la construcción de confianza y legitimidad requerida para el funcionamiento de las democracias. Por tanto, preguntarse hoy por la participación ciudadana es cuestionar cómo se da la comunicación entre gobiernos y ciudadanía en tiempos digitales $\mathrm{y}$ en un contexto de desafección política.

Global Media Journal México 17(32), enero - junio 2020, pp. 19-41. 
A partir de esta pregunta, el análisis de los compromisos de gobierno abierto expuesto anteriormente cuestiona el para qué de la comunicación, al caracterizar los compromisos con relación a los ejes de transparencia, participación, colaboración y gestión pública. Asimismo, cuestiona el modelo de comunicación al describir los niveles de participación, y se pregunta por la forma en la que se da la comunicación desde el análisis de las plataformas tecnológicas que se proponen, con sus funcionalidades y posibilidades de interacción para la ciudadanía.

Aunque los resultados sugieren que aún estamos en la primera etapa del proceso de implementación de un modelo de gobierno abierto, esta etapa se caracteriza porque los compromisos se ubican especialmente en el ámbito de la transparencia y se limitan a acciones informativas. Las promesas de participación ciudadana mediante las TICs aún están limitadas por la falta de compromisos más centrados en la colaboración y el involucramiento de la ciudadanía.

Se puede observar un giro de la perspectiva de una comunicación unidireccional a una más participativa en algunos aspectos, como si empezara a plantearse la comunicación y el diálogo entre la ciudadanía y los gobiernos desde el diseño de las plataformas tecnológicas, no solo en su puesta en marcha. También, las TICs comienzan a utilizarse como una herramienta que se incluye en estrategias para fomentar la participación ciudadana en temas específicos (por ejemplo, niñez y género), mediante la combinación de acciones virtuales y presenciales. Y, por último, las tecnologías han de vincularse al ciclo de las políticas públicas, superando su uso solo para la rendición de cuentas y la gestión de trámites y servicios.

En los compromisos, la participación ciudadana se manifiesta en diversas acciones como lo son el diseño de plataformas, el uso de espacios virtuales como las redes sociales para la colaboración, y la promoción de la participación ciudadana en el ciclo de las políticas públicas mediante encuentros presenciales e instrumentos virtuales.

En cuanto a las plataformas para la participación ciudadana, aumenta la oferta de portales web de datos abiertos y plataformas para informar, trabajar de forma conjunta y el monitoreo de políticas públicas. Los sistemas de información y bases de datos, no solo resuelven la gestión interna y comienzan a publicar información relacionada con presupuestos, contrataciones y obras públicas, así como registros de personas beneficiarias de políticas públicas $\mathrm{y}$ directorios de instituciones $\mathrm{y}$ organizaciones.

La inserción de datos abiertos en el debate le agrega complejidad a la participación; para participar la ciudadanía necesita estar informada, pero ahora la información no solo debe ser pertinente, también debe estar en formato abierto para facilitar su reutilización y generar mayor valor. En otras palabras, el dato ha de tener formato abierto para que su reutilización 
potencie la participación y el involucramiento.

El modelo de gobierno abierto ha servido para promover iniciativas que apuntan a mejorar la interacción entre gobiernos y ciudadanía. De acuerdo con el análisis, en este proceso las TICs siguen siendo protagonistas, pero queda pendiente la cuestión de cómo aprovecharlas para superar la idea del gobierno electrónico, centrada en la noción del usuario de servicios, para pasar a la perspectiva de gobierno abierto, en el que la ciudadanía sería parte de la gestión y de la resolución de los problemas públicos. La investigación muestra que las promesas inconclusas del gobierno electrónico ahora han sido integradas al gobierno abierto, sin cuestionar el lugar de la ciudadanía en la relación comunicativa.

En términos de comunicación, el reto de los gobiernos frente al gobierno abierto tiene que ver con transformar el modelo de comunicación tradicional, que aún permea las iniciativas tecnológicas que se incluyen en los compromisos de gobierno abierto, y con repensar el desarrollo de TICs para facilitar la participación de la ciudadana en todos los niveles, desde la deliberación hasta la toma de decisiones, inspirándose en las prácticas que propician el mundo digital y la cultura participativa.

\section{Referencias bibliográficas}

Alianza para el Gobierno Abierto México. (2011). $1^{\circ}$ Plan de Acción de México.. https://observatorioplanificacion.cepal.org/sites/default/files/plan/files/1_Mexico_Action_Plan_0.pdf

Alianza para el Gobierno Abierto México. (2013). $2^{\circ}$ Plan de Acción 2013- 2015 México. https://observatorioplanificacion.cepal.org/sites/default/files/plan/files/2_pa_aga_2015\%20\%281\% 29.pdf

Alianza para el Gobierno Abierto México. (2016). $3^{\circ}$ Plan de Acción de 2016 - 2018, Tercer Plan de Acción Nacional de México. https://drive.google.com/open?id=1iwYxRf8YJpZXHtUfFyVAfqrjU8RvSbhS

Alianza para el Gobierno Abierto México. (2019). $4^{\circ}$ Plan de Acción 2019-2021 de México. https://drive.google.com/open?id=1OTGV4biMJscNF67Nr1cNKY61C7H1qujz

Animal Político. (2017, 23 de mayo). Sociedad civil denuncia espionaje y rompe con la Alianza por el Gobierno Abierto. Animal Politico. http://www.animalpolitico.com/2017/05/espionaje-alianzagobierno-abierto/

Borrmann, R. (2017, 14-17 de noviembre). Avances de Argentina en politicas de gobierno abierto [Artículo presentado]. XXII Congreso Internacional del CLAD sobre la Reforma del Estado y de la Administración Pública, Madrid, España. http://www2.congreso.gob.pe/sicr/cendocbib/con5_uibd.nsf/FAE8F8F14804E4D0052582330073F

Global Media Journal México 17(32), enero - junio 2020, pp. 19-41. 
70B/\$FILE/borrmrud.pdf

Castells, M. (2008). Comunicación, poder y contrapoder en la sociedad red (I): Los medios y la política. Telos, (74), 13-24. $\quad$ http://www.psiaudiovisuales.com.ar/wpcontent/uploads/Castells_Comunicacion-poder-y-contrapoder-en-la-sociedad-red.pdf

Comisión Nacional de Gobierno Abierto de Costa Rica. (2015, 2 de diciembre). Eje de Transparencia y Acceso a la Información. http:/gobiernoabierto.go.cr/eje-de-transparencia-y-acceso-a-lainformacion/

Concha, G., y Naser, A. (2012). Datos abiertos: un nuevo desafio para los gobiernos de la región. CEPAL / @LIS http://repositorio.cepal.org/bitstream/handle/11362/3969/1/S2012004_es.pdf

Concha, G., y Naser, A. (2012). El desafio hacia el gobierno abierto en la hora de la igualdad. CEPAL / @LIS http://www.datosabiertos.gob.ec/wpcontent/uploads/downloads/2016/08/El_desafio_hacia_el_gobierno_abierto.pdf

Corojan, A., y Domínguez, E. C. (2011). Gobierno abierto: alcance e implicaciones. Madrid: Fundación Ideas.

en: https://ialnet.unirioja.es/servlet/libro?codigo $=572551 \&$ info=resumen\&idioma $=$ SPA

Cruz-Rubio, C. N. (2015). Hacia el gobierno abierto: Una caja de herramientas. Departamento para la Gestión Pública Efectiva-OEA. http://www.gigapp.org/administrator/components/com_jresearch/files/publications/FINAL\%20Caj a\%20de\%20Herramientas.pdf

Damasco, D. P. (2017, 31 de enero). ¿Quién es quién en datos abiertos en América Latina? - Distintas Latitudes. https://distintaslatitudes.net/mapa-datos-abiertos-america-latina

Gobierno abierto en México. (2017, 28 de febrero). El Universal. http://www.eluniversal.com.mx/blogs/observatorio-nacional-ciudadano/2017/02/28/gobiernoabierto-en-mexico

Gómez Mendoza, M. A. (2012, 20 de mayo). Análisis de contenido cualitativo y cuantitativo: definición, clasificación y metodología. Metodologiaecs. https://metodologiaecs.wordpress.com/2012/05/20/analisis-de-contenido-cualitativo-y-cuantitativo

Jenkins, H., Ford, S., y Green, J. (2013). Cultura transmedia: la creación de contenido y valor en una cultura en red. Gedisa.

Krippendorff, K. (1997). Metodología de análisis de contenido. Paidós.

Martínez, N., Tucho, F., y García de Madariaga, J. M. (2005). Democracia digital: nuevos medios y participación ciudadana. Experiencias en la red de la población inmigrante en España. Portularia: Revista de Trabajo Social, (5), 21-34. http://dialnet.unirioja.es/servlet/articulo?codigo=2134228

Global Media Journal México 17(32), enero - junio 2020, pp. 19-41. 
Mattelart, A. (2001). Historia de la sociedad de la información. Paidós. https:/octavioislas.files.wordpress.com/2014/08/mattelart-armand-historia-de-la-sociedad-de-lainformacion1.pdf

McLuhan, M., y Nevitt, B. (1972). Take today: The executive as dropout. Harcourt Brace Jovanovich.

Miniwatts Marketing Group. (2018). Internet world stats usage and population statisTIC [versión de 4 de marzo de 2019]. http://www.internetworldstats.com/

Núcleo de la Sociedad Civil para el Gobierno Abierto en México. (17 de julio de 2018). Carta abierta de sociedad civil mexicana al Comité Directivo de la AGA - Núcleo de la Sociedad Civil para el Gobierno Abierto en México. https://gobiernoabiertomx.org/blog/2018/07/17/carta-abierta-desociedad-civil-mexicana-al-comite-directivo-de-la-aga/

OGP Argentina. (2018). Sobre la evaluación de los avances en la implementación de los compromisos del 3er Plan de Acción del Estado argentino en el marco de la Alianza para el Gobierno Abierto OGP Argentina. https://ogpargentina.org/2018/10/25/sobre-la-evaluacion-de-los-avances-en-laimplementacion-de-los-compromisos-del-3er-plan-de-accion-del-estado-argentino-en-el-marco-dela-alianza-para-el-gobierno-abierto/

Petrizzo-Páez, Á.-M. (2005). Participación ciudadana y tecnologías de la información y la comunicación: hacia una administración pública relacional. Nueva Sociedad, (195), 88-101. https://nuso.org/media/articles/downloads/3241_1.pdf

Presidencia de la Nación de Argentina. (2013). Plan de Acción de la República de Argentina 2013. http://observatorioplanificacion.cepal.org/sites/default/files/plan/files/1_ARGENTINA_20132015_0.pdf

Presidencia de la Nación de Argentina. (2015). 2do Plan de Acción Nacional de Gobierno Abierto de la República de Argentina 2015-2017. http://observatorioplanificacion.cepal.org/sites/default/files/plan/files/1_ARGENTINA_20132015_0.pdf

Presidencia de la Nación Argentina. (2017). 3er Plan de Acción de Gobierno Abierto de la República de $\begin{array}{lll}\text { Argentina } & \text { 2017-2019. } & \text { Recuperado }\end{array}$ http://observatorioplanificacion.cepal.org/sites/default/files/plan/files/3_Argentina_ActionPlan_2017-2019.pdf

Presidencia República de Costa Rica (2013). Plan de Acción Costa Rica 2013-2014. https://observatorioplanificacion.cepal.org/sites/default/files/plan/files/1_Costa_Rica_20132015.pdf

Global Media Journal México 17(32), enero - junio 2020, pp. 19-41. 
Presidencia República de Costa Rica. (2015). 2do Plan de Acción ante la Alianza por un Gobierno Abierto 2015-2017. https://observatorioplanificacion.cepal.org/sites/default/files/plan/files/2_Segundo\%20Plan\%20de \%20Acci\%C3\%B3n\%20Costa\%20Rica\%20\%281\%29.pdf

Presidencia República de Costa Rica. (2017). 3er Plan de Acción de la Alianza para un Gobierno Abierto 2017-2019. https://app.box.com/s/bixgocf3k86c56wbzfp3zib3t8hgcwjv

Sánchez, M., y Ibar, R. (2015). Convergencia e interacción en los nuevos medios: tipologías de prosumidores entre los estudiantes universitarios. Communication \& Society, 28(2), 87-99. https://doi.org/10.15581/003.28.2.sp.87-99

Ure, M. (2015). La comunicación de la administración pública en las redes sociales: los casos de las ciudades de Buenos Aires y Bolonia. Palabra Clave, 19(1), 240-270. https://doi.org/10.5294/pacla.2016.19.1.10

Vilches, C. (2016, mayo 19). Biblioguias: de gobierno abierto a Estado abierto: Planes de acción regional. https://biblioguias.cepal.org/EstadoAbierto/planes

Wikipedia. (2018). Anexo: Países por número de teléfonos móviles. https://es.wikipedia.org/w/index.php?title=Anexo:Países_por_número_de_teléfonos_móviles\&oldi $\mathrm{d}=100189542$

Global Media Journal México 17(32), enero - junio 2020, pp. 19-41. 